\title{
Discrete design models of buildings at dynamic calculation
}

\author{
Vladimir Mondrus ${ }^{1}$, and Stanislav Shutovskiy ${ }^{{ }^{*}}$ \\ ${ }^{1}$ Moscow State University of Civil Engineering, Yaroslavskoe shosse, 26, 129337, Moscow, Russia
}

\begin{abstract}
In the article settlement models of the buildings and constructions having periodic (regular) structure with a masses concentrated in nods are considered. Analysis method for finding modal frequencies of free fluctuations for step-periodic buildings are shown.
\end{abstract}

Let's consider design models of the multi-storey buildings and constructions having step and periodic structure. It is possible to carry buildings with the sizes of loadings and sections of load-carrying structures changing on height to such constructions. Changes on building height usually happen in such a way that sections of elements of designs and the size of loadings remain constants throughout several floors. Design models of such buildings and constructions can be presented in the form shown in fig. 1a.

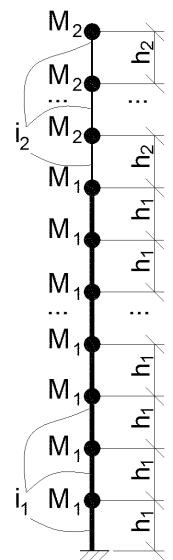

a)

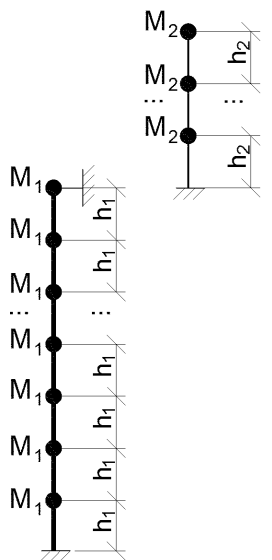

б)

Fig. 1. Example of step periodic structure.

Calculation of a step and periodic design model can be reduced to calculation of separate components of periodic sites, having used a method of movements [3]. At the same time the main system should be formed by introduction of additional communications only in places of interfaces of periodic sites (fig. 1b). The received complex main system

\footnotetext{
* Corresponding author: shutovskii@mail.ru
} 
consists of multi-storey periodic sites, calculation of each of which can be executed by means of the receptions used for calculation of the periodic models which are not changing stepwise on height [2].

Let's use the following designations: unknown harmonious movements in the direction of the entered communications

$$
-Y_{1}(t)=Y_{1}(t) \exp (i \omega t), Y_{2}(t)=Y_{2}(t) \exp (i \omega t), \ldots, Y_{l}(t)=Y_{l}(t) \exp (i \omega t)
$$

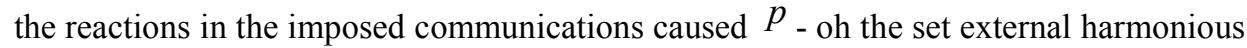
loading with a frequency $\omega$,

$$
-R_{1 p}(t)=R_{1 p}(t) \exp (i \omega t), R_{2 p}(t)=R_{2 p}(t) \exp (i \omega t), \ldots, R_{l p}(t)=R_{l p}(t) \exp (i \omega t)
$$

the constraint force $n^{\prime}$ caused by single harmonious movement of communication $m^{\prime}$ $\left(Y_{m^{\prime}}(t)=1 \cdot \exp (i \omega t)\right)$

$$
r_{n^{\prime} m^{\prime}}(t)=r_{n^{\prime} m^{\prime}}(t) \exp (i \omega t) \text {, }
$$

The equality condition to zero total reaction in $n$ - oh the imposed communication (the canonical equation of a method of movements) has an appearance

$$
r_{n^{\prime} 1} Y_{1}+r_{n^{\prime} 2} Y_{2}+\ldots+r_{n^{\prime} l} Y_{l}+R_{n^{\prime} p}=0 \text {. }
$$

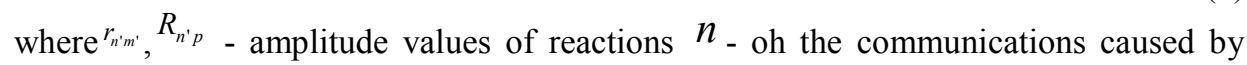
single harmonious movement of communication $m$ and $p$ - oh the set external harmonious loading; ${ }_{n^{\prime}}$ - amplitude value of unknown harmonious movement in the direction $n^{\prime}$ of communication.

$\operatorname{At}^{n}=1,2, \ldots, l$ we will receive system $l$ of the canonical equations, solving which, it is found amplitude values of unknown movements

$Y_{1}, Y_{2}, \ldots, Y_{l}$.

As the complex main system consists of multi-storey periodic sites, creation of single diagrams of movements of the main system from amplitude values of single harmonious movements of support, and also from the set loading, comes easy.

If the design model is affected by an arbitrary force $F(t)$, then this force can be decomposed on any interval of time in a row by Fourier. The design model is calculated on everyone composed a row separately, and then results of calculations are summed up.

Free fluctuations of step and periodic design models are investigated by means of the canonical equations (1) without absolute terms

$$
r_{n^{\prime} 1} Y_{1}+r_{n^{\prime} 2} Y_{2}+\ldots+r_{n^{\prime} l} Y_{l}=0 .
$$

As these equations are homogeneous, for obtaining the values other than zero, it is necessary determinant from coefficients at unknown in these equations to turn into zero

$$
D=\left|\begin{array}{cccc}
r_{11} & r_{12} & \ldots & r_{1 l} \\
r_{21} & r_{22} & \ldots & r_{2 l} \\
\ldots & \ldots & \ldots & \ldots \\
r_{l 1} & r_{l 2} & \ldots & r_{l l}
\end{array}\right|=0
$$

Opening determinant, we will receive the equation of frequencies concerning unknown $^{\omega_{j}}$. All solutions of an equation will be free oscillation frequencies of the studied design model. 


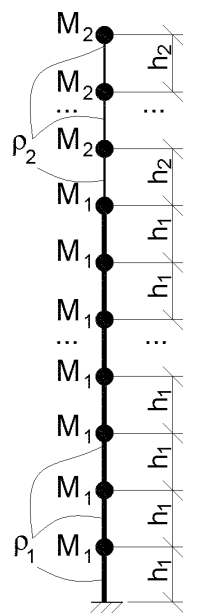

a)

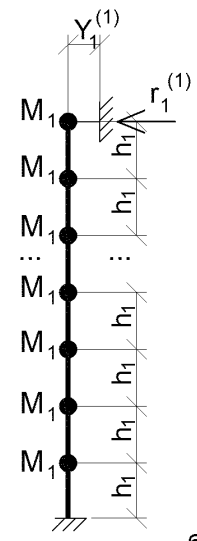

б)

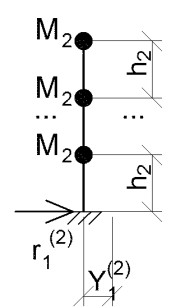

Fig. 2. Design model.

Let's trace the sequence of determination of frequencies and forms of free fluctuations on the example of the building consisting of two any periodic sites. The design model of this construction is given in fig. 2a. The main system is shown in fig. $2 b$. The corresponding canonical equation has an appearance

$$
r_{11} Y_{1}=0
$$

where $^{r_{11}}$ - amplitude value of reaction of the entered communication caused by single harmonious movement of the same communication; $Y_{1}$ - amplitude value of the unknown of movements in the direction of the entered communication.

Equation of frequencies

$$
r_{11}=0
$$

Single harmonious movement of the entered communication, and also the reactions caused by it are shown in fig. 2 b.

It is obvious that

$$
r_{11}=r_{11}^{(1)}+r_{11}^{(2)}
$$

where $^{r_{11}^{(1)}}$ and ${ }^{r_{11}^{(2)}}$ - respectively amplitude values of reactions of the imposed communication for the lower and upper sites.

Thus

$$
r_{11}=\rho\left(1+\frac{C_{k_{1}-2}^{1}\left(\xi_{1}\right)}{C_{k_{1}-1}^{1}\left(\xi_{1}\right)}\right)-M_{1} \omega^{2}+\frac{2 \rho_{2}\left(1+\xi_{2}\right) C_{k_{1}-2}^{1}\left(\xi_{2}\right)}{C_{k_{2}}^{1}\left(\xi_{2}\right)+C_{k_{2}-1}^{1}\left(\xi_{1}\right)}=0
$$

from where

$$
\frac{2 \rho_{2}\left(1+\xi_{2}\right) C_{k_{1}-2}^{1}\left(\xi_{2}\right)}{\rho_{1}\left[C_{k_{2}}^{1}\left(\xi_{2}\right)+C_{k_{2}-1}^{1}\left(\xi_{1}\right)\right]}=1+2 \xi_{1}-\frac{C_{k_{1}-2}^{1}\left(\xi_{1}\right)}{C_{k_{1}-1}^{1}\left(\xi_{1}\right)}
$$

where $\xi_{1}=\frac{M_{1} \omega^{2}}{2 \rho_{1}}-1 \xi_{2}=\frac{M_{2} \omega^{2}}{2 \rho_{2}}-1$.

The solution of this equation $\omega$ rather more simply than everything, apparently, can be found in the graphic-analytical way. When determining numerical values of both parts of equality it is possible to use tables or schedules of polynoms of Gegenbauer [6,7]. 
For the solution of specific objectives it is possible to simplify the equation (8) a little if to present it in a trigonometrical form. At the same time the type of the equation depends on the size of parameters $\xi_{1}$ and $\xi_{2}$.

For any form of free fluctuations of movement of mass of the lower and upper sites are defined by expressions

$$
y_{n_{1} j}(t)=y_{n_{1} j}(t) \exp \left[i\left(\omega_{j} t-\varphi_{j}\right)\right] y_{n_{2} j}(t)=y_{n_{2} j}(t) \exp \left[i\left(\omega_{j} t-\varphi_{j}\right)\right] .
$$

It is easy to see that the condition of lack of a gap in movements in the place of contact of sites is satisfied in the most general case since.

$$
y_{\left(n_{1}=k_{1}\right) j}(t)=y_{\left(n_{2}=0\right) j}(t)=y_{j}(t) \exp \left[i\left(\omega_{j} t-\varphi_{j}\right)\right] .
$$

The common decision for free fluctuations is described by expressions

$$
\begin{aligned}
& y_{n_{1} j}(t)=(-1)^{k_{1}+n_{1}} \sum_{j=1}^{k_{1}+k_{2}} \frac{C_{n_{1}-1}^{1}\left(\xi_{1 j}\right)}{C_{k_{1}-1}^{1}\left(\xi_{1 j}\right)} Y_{j} \exp \left[i\left(\omega_{j} t-\varphi_{i}\right)\right], \\
& y_{n_{2} j}(t)=(-1)^{n_{2}} \sum_{j=1}^{k_{1}+k_{2}} \frac{C_{k_{1}-n_{2}}^{1}\left(\xi_{2 j}\right)+C_{k_{2}-n_{2}-1}^{1}\left(\xi_{2 j}\right)}{C_{k_{2}}^{1}\left(\xi_{2 j}\right)+C_{k_{2}-1}^{1}\left(\xi_{2 j}\right)} Y_{j} \exp \left[i\left(\omega_{j} t-\varphi_{j}\right)\right],
\end{aligned}
$$

In total $2\left(k_{1}+k_{2}\right)$ arbitrary constants of a look $Y_{j} \varphi_{j}$ it is also possible to define, having used $^{2\left(k_{1}+k_{2}\right)}$ initial conditions of donation of each weight at $t=0$. Such initial conditions are movements of masses $y_{n_{1}}(0), y_{n_{2}}(0)$ and their speeds $\dot{y}_{n_{1}}(0) \dot{y}_{n_{2}}(0)$.

Efforts in the communications connecting two consistently located masses lower (upper) sites are determined by formulas

$$
\begin{aligned}
& \left.F_{n_{1}\left(n_{1}-1\right)}(t)=\rho_{1} \mid y_{n_{1}}(t)-y_{n_{1}-1}(t)\right]= \\
& =(-1)^{k_{1}+n_{1}} \rho_{1} \sum_{j=1}^{k_{1}+k_{2}} \frac{C_{n_{1}-1}^{1}\left(\xi_{1 j}\right)+C_{n_{1}-2}^{1}\left(\xi_{1 j}\right)}{C_{k_{1}-1}^{1}\left(\xi_{1 j}\right)} Y_{j} \exp \left[i\left(\omega_{j} t-\varphi_{i}\right)\right] \\
& F_{n_{2}\left(n_{2}-1\right)}(t)=\rho_{2}\left[y_{n_{2}}(t)-y_{n_{2}-1}(t)\right]= \\
& =(-1)^{n_{2}} \sum_{j=1}^{k_{1}+k_{2}} \frac{C_{k_{1}-n_{2}-1}^{1}\left(\xi_{2 j}\right)+C_{k_{2}-n_{2}+1}^{1}\left(\xi_{2 j}\right)}{C_{k_{2}}^{1}\left(\xi_{2 j}\right)+C_{k_{2}-1}^{1}\left(\xi_{2 j}\right)} Y_{j} \exp \left[i\left(\omega_{j} t-\varphi_{j}\right)\right]
\end{aligned}
$$

The approach to a research of free and forced vibration of design models stated below in the form of chains of oscillators with a finite number of the masses (Fig. 3) can be used as for studying of the fluctuations caused by translational motions of masses along any of axes $0 x 0 y 0 z$, and for studying of fluctuations in the course of which masses makes torsional oscillation around the same axes. For an illustration in the present article we will consider fluctuations in the course of which masses moves only progressively along an axis $0 z$. 


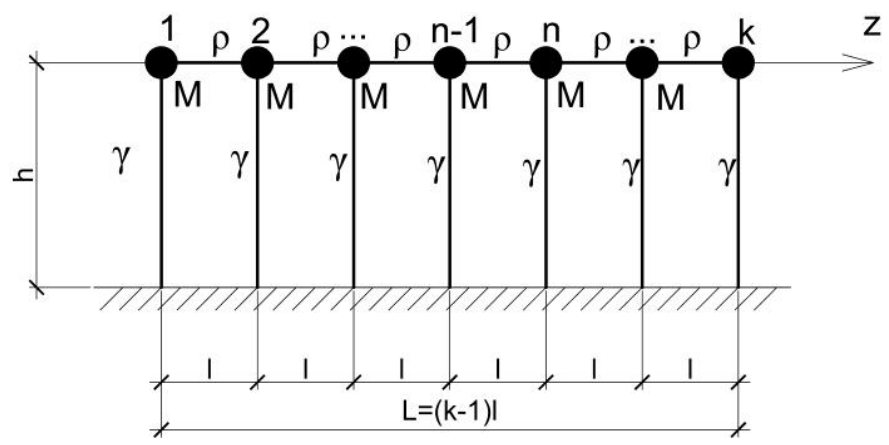

Fig. 3. Model one-storied extended building.

Let's consider free fluctuations of the design model represented in fig. 3. Let's write down the allowing system of differential equations in the following look

$$
\begin{aligned}
& M \ddot{z}_{1}+(\rho+\gamma) z_{1}-\rho z_{2}=0, \\
& \ldots \\
& M \ddot{z}_{n}-\rho z_{n-1}+(2 \rho+\gamma) z_{n}-\rho z_{n+1}=0, \\
& \cdots \\
& M \ddot{z}_{k}-\rho z_{k-1}+(\rho+\gamma) z_{k}=0,
\end{aligned}
$$

The solution of this system can be found in a look

$$
z(t)=A_{n} \exp [i(\omega t-\varphi)] n=1,2, \ldots, k
$$

After some transformations we come to a system of equations

$$
\begin{aligned}
& (2 \xi+1) A_{1}+A_{2}=0, \\
& \cdots \\
& A_{n-1}+2 \xi A_{n}+A_{n+1}=0, \\
& \cdots \\
& A_{k-1}+(2 \xi+1) A_{k}=0,
\end{aligned}
$$

where

$$
\frac{M \omega^{2}-2 \rho-\gamma}{\rho}=2 \xi
$$

That the system (15) had a nontrivial solution, the equality condition to zero its determinant has to be satisfied

$$
D=C_{k}^{1}(\xi)+2 C_{k-1}^{1}(\xi)+2 C_{k-2}^{1}(\xi)=2(1+\xi) C_{k-1}^{1}(\xi)=0
$$
[4].

where expressions $C_{k}^{1}(\xi), C_{k-1}^{1}(\xi), C_{k-2}^{1}(\xi)$ - Gegenbauer's polynoms of the first degree

This equation has $k$ roots, one of which is equal,

$$
\xi_{0}=-1
$$

And the others $k-1$ of roots are defined from a condition

$$
C_{k-1}^{1}(\xi)=0
$$

The polynom $C_{k-1}^{1}(\xi)$ has $k-1$ zero in an interval $(-1 ;+1)$ at values of an argument 


$$
\xi_{j}=\cos \theta_{j}=\cos \frac{j \pi}{k}, \text { where } j=1,2, \ldots, k
$$

Expressions for frequencies of eigentones of model will look as follows

$$
\omega_{0}=\sqrt{\frac{\gamma}{M}} \omega_{j}^{2}=\frac{4 \rho}{M} \cos ^{2} \frac{j \pi}{2 k}+\frac{\gamma}{M}, \text { where } j=1,2, \ldots, k
$$

It is also possible to write down the second of formulas (21) in a look at which to bigger number $\tilde{J}$ there will correspond big frequency:

$$
\omega_{\tilde{J}}^{2}=\frac{4 \rho}{M} \sin ^{2} \frac{\widetilde{J} \pi}{2 k}+\frac{\gamma}{M} \text { where } \widetilde{J}=k-j
$$

Frequency $\omega_{0}$ answers such movement of a design model when all masses fluctuate in a phase with an identical amplitude $A_{10}=A_{20}=\ldots=A_{k 0}=A_{0}$.

For each free oscillation frequency there is a form of fluctuations corresponding to it. It is defined as follows:

$$
z_{n j}(t)=A_{n j} \exp \left[i\left(\omega_{j} t-\varphi_{j}\right)\right]
$$

Having carried out some transformations, we receive expression for

$$
\begin{aligned}
& z_{n 0}(t)=A_{0} \cos \left(\sqrt{\frac{\gamma}{M}} t-\varphi_{0}\right), \\
& z_{\tilde{n} \mathfrak{j}}(t)=(-1)^{k+n} A_{\widetilde{\jmath}} \sin \frac{(2 n-1) \pi(k-\widetilde{\jmath})}{2 k} \cos \left(t \sqrt{\frac{4 \rho}{M} \sin ^{2} \frac{\widetilde{\jmath} \pi}{2 k}+\frac{\gamma}{M}}-\varphi_{\widetilde{\jmath}}\right),
\end{aligned}
$$

where $^{A_{\widetilde{\jmath}}}$ and $\varphi_{\widetilde{\jmath}}$ - arbitrary constants.

Generally the movement $n_{-}$oh the mass of a design model is described by the following formula:

$$
\begin{aligned}
& z_{n}(t)=A_{0} \cos \left(\sqrt{\frac{\gamma}{M}} t-\varphi_{0}\right)+ \\
& +(-1)^{k+n} \sum_{\tilde{j}=1}^{k-1} A_{\tilde{j}} \sin \frac{(2 n-1) \pi(k-\widetilde{j})}{2 k} \cos \left(t \sqrt{\frac{4 \rho}{M} \sin ^{2} \frac{\widetilde{j} \pi}{2 k}+\frac{\gamma}{M}}-\varphi_{\tilde{j}}\right),
\end{aligned}
$$

Let's consider fluctuation of the basis of several racks of a design model. Let's assume that $\widetilde{k}(\widetilde{k} \leq k)$ racks fluctuations under any non-periodic law at the same time make $\bar{z}_{m}(t)$. The system of equations will look as follows

$$
\begin{aligned}
& M \ddot{z}_{1}+(\rho+\gamma) z_{1}-\rho z_{2}=\gamma \int_{-\infty}^{+\infty} G_{1}(\omega) \exp (i \omega t) d \omega, \\
& \ldots \\
& M \ddot{z}_{m-1}-\rho z_{m-2}+(2 \rho+\gamma) z_{m-1}-\rho z_{m}=0, \\
& M \ddot{z}_{m}-\rho z_{m-1}+(2 \rho+\gamma) z_{m}-\rho z_{m+1}=\gamma \int_{-\infty}^{+\infty} G_{m}(\omega) \exp (i \omega t) d \omega, \\
& \ldots \\
& \ddot{z}_{k}-\rho z_{k-1}+(\rho+\gamma) z_{k}=\gamma \int_{-\infty}^{+\infty} G_{k}(\omega) \exp (i \omega t) d \omega .
\end{aligned}
$$


At fluctuations under the harmonious law the column of absolute terms will look as follows

$$
\mid \begin{aligned}
& \gamma z_{1} \exp \left[i\left(\omega_{1} t-\varphi_{1}\right)\right] \\
& \gamma z_{m} \exp \left[i\left(\omega_{m} t-\varphi_{m}\right)\right] \\
& \gamma z_{k} \exp \left[i\left(\omega_{k} t-\varphi_{k}\right)\right]
\end{aligned}
$$

Let's consider a case of distribution of a wave under a construction. Let's accept that the wave is harmonious

$$
\bar{z}(t)=z \exp (i \omega t)
$$

$z$ - wave amplitude, $\omega$ - wave frequency.

Fluctuations of each rack are dephased concerning fluctuations of previous and the

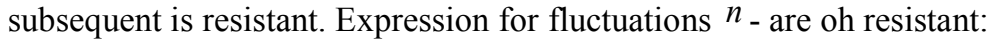

$$
\bar{z}_{m}(t)=z \exp \left[i\left(\omega t-\varphi_{m}\right)\right]
$$

Phase displacement concerning the first rack is defined as:

$$
\varphi_{m}=\frac{\omega l(m-1)}{c}
$$

$l$ - a spacing of props, ${ }^{c}$ - the speed of distribution of a wave.

Expression for movements $n_{\text {- }}$ oh masses when passing under the building of a harmonious wave:

$$
z_{n}(t)=\sum_{m=1}^{k} z_{n m}(t)=\sum_{m=1}^{k} A_{n m} \exp \left[i\left(\omega t-\varphi_{m}\right)\right]
$$

Sizes $A_{n m}$ are determined by a formula (12).

Let's make a research of the specific building which rated scheme is represented in fig. 4, when passing under it a harmonious wave of a look $\bar{z}(t)=z \sin (\omega t)$. Let's accept wave parameters $c=7 \cdot 10^{4} \mathrm{~cm} / \mathrm{c} T=0.4 c \omega=15.7 \Gamma u \lambda=c T=2.8 \cdot 10^{4} \mathrm{~cm}$.

Zhestkostny characteristics of model $\gamma=10^{3} \kappa 2 / c M \rho=10^{4} \kappa 2 / c M$, the weight concentrated in a node $M=100 \kappa 2$.

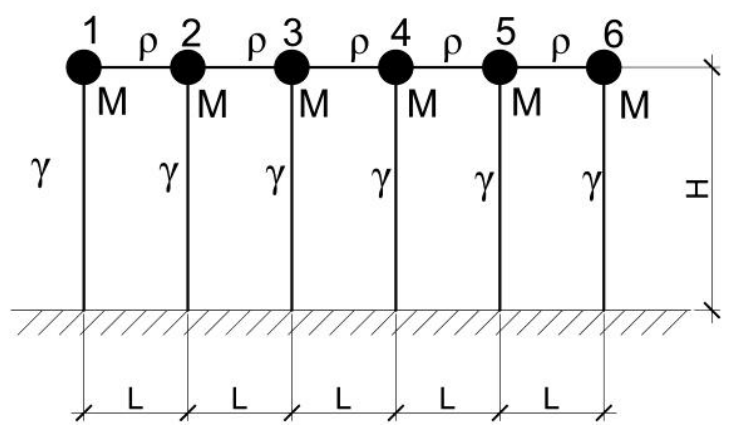

Fig. 4. Model one-storied extended building.

We will determine frequencies of eigentones of model by expressions (21) and (22). Distribution of amplitudes for each of forms - according to (24).

When passing under the building of a wave of movement of masses are on a formula

$$
z_{n}(t)=\sum_{m=1}^{6} A_{n m} \sin \left(\omega t-\varphi_{m}\right)
$$


$A_{n m}$ - amplitude values of movements of weight $n$ at fluctuations of the basis of a $\operatorname{rack}^{m}$ with frequency $\omega$. Phase displacements $\varphi_{m}$ are determined by a formula $\varphi_{m}=\frac{\omega \cdot L}{c}(m-1) . m$ - number of weight.

The calculated movements $z_{n}(t)$ in a surface appearance of movements of masses are shown in fig. 5 .

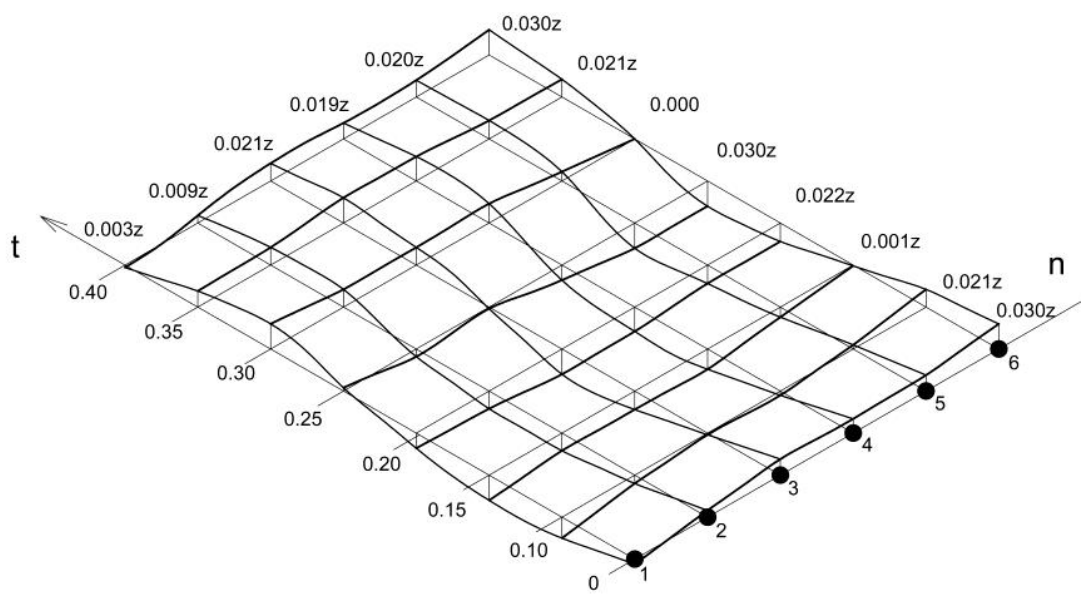

Fig. 5. Calculated movements of masses.

As the considered interval of time $T$ matches the period extending the waves given on the drawing results completely describe movements of a design model and longwise, and on time.

\section{References}

1. L. Brilluen, M. Parody, Distribution of waves in periodic structures. (SILT, Moscow, 1959)

2. B. P. Wolfson, Researches on the theory of constructions, under. edition of the prof. B. G. Korenev, member correspondent of Academy of Sciences of the USSR I. M. Rabinovich, prof. A. F. Smirnov, XYII (1969).

3. B.P. Volfson. About wave propagation in models of buildings and constructures with internal friction. Journal of "Structural mechanics and analysis of constructions" No. 5, (1971)

4. B. P. Wolfson, Researches on the theory of constructions, under. edition of the prof. B. G. Korenev, member correspondent of Academy of Sciences of the USSR I. M. Rabinovich, prof. A. F. Smirnov, XIX (1972)

5. M. A. Dashevsky, Works TsNIISK of V. A. Kucherenko, 43 (1975)

6. A. F. Nikiforov, V. B. Uvarov. Special functions of the mathematical physics. Intellekt (2007)

7. G. Szegö. Orthogonal polynomials. (The state publishing house of the physical and mathematical literature, Moscow, 1962)

8. P.K. Suetin Classical orthogonal polynomials, (Fizmatlit, Moscow, 2007) 